University of South Carolina

Scholar Commons

$9-15-2008$

\title{
Spin Memristive Systems: Spin Memory Effects in Semiconductor Spintronics
}

Yuriy V. Pershin Dr

University of South Carolina - Columbia, pershin@physics.sc.edu

M. Di Ventra

Follow this and additional works at: https://scholarcommons.sc.edu/phys_facpub

Part of the Physics Commons

Publication Info

Published in Physical Review B, ed. Gene D. Sprouse, Volume 78, Issue 11, 2008, pages

113309-1-113309-4.

Pershin, Y. V., \& Di Ventra, M. (2008). Spin memristive systems: Spin memory effects in semiconductor spintronics. Physical Review B, 78(11), 113309-1 - 113309-4. DOI: 10.1103/PhysRevB.78.113309

(C) Physical Review B, 2008, American Physical Society

This Article is brought to you by the Physics and Astronomy, Department of at Scholar Commons. It has been accepted for inclusion in Faculty Publications by an authorized administrator of Scholar Commons. For more information, please contact digres@mailbox.sc.edu. 


\title{
Spin memristive systems: Spin memory effects in semiconductor spintronics
}

\author{
Yu. V. Pershin and M. Di Ventra \\ Department of Physics, University of California-San Diego, La Jolla, California 92093-0319, USA
}

(Received 28 August 2008; published 23 September 2008)

\begin{abstract}
Recently, in addition to the well-known resistor, capacitor, and inductor, a fourth passive circuit element, named memristor, has been identified following theoretical predictions. The model example used in such case consisted in a nanoscale system with coupled ionic and electronic transport. Here, we discuss a system whose memristive behavior is based entirely on the electron-spin degree of freedom, which allows for a more convenient control than the ionic transport in nanostructures. An analysis of time-dependent spin transport at a semiconductor/ferromagnet junction provides a direct evidence of memristive behavior. Our scheme is fundamentally different from previously discussed schemes of memristive systems and broadens the possible range of applications of semiconductor spintronics.
\end{abstract}

DOI: 10.1103/PhysRevB.78.113309

PACS number(s): 73.23.Hk, 72.25.Dc, 72.25.Mk

In 1971 Leon Chua, ${ }^{1}$ analyzing mathematical relations between pairs of fundamental circuit variables, proposed a fourth two-terminal circuit element characterized by a relationship between the charge and the flux. He called that element a memristor (or memory resistor). Five years later, Chua and $\mathrm{Kang}^{2}$ introduced a more general class of twoterminal devices: memristive systems. If $w$ denotes a set of $n$-state variables describing the internal state of the system, an $n$ th-order current-controlled memristive system is described by the equations

$$
\begin{gathered}
V=R(w, i, t) i, \\
\dot{w}=f(w, i, t),
\end{gathered}
$$

where $V$ and $i$ denote the voltage and current and $R$ is a generalized resistance. The equation for a memristor is a particular case of Eqs. (1) and (2), when $R$ depends only on charge, namely,

$$
V=R[q(t)] i .
$$

Although several experimental systems were suggested to behave as memristive systems, ${ }^{2}$ the real interest in these devices resurfaced only recently when a group of scientists from Hewlett Packard identified a specific experimental realization of memristor. ${ }^{3}$ In their scheme, a memory effect is achieved in solid-state thin film two-terminal devices. In that case, the memristive behavior is based on the coupling between transport of atomic degrees of freedom (e.g., oxygen vacancies acting as mobile dopants defining the internal state of the device) and of electrons. The electron current flowing through such a device dynamically changes the internal state of the latter, which, in turn, influences the electron transport in a nonlinear way.

Ionic transport, however, relies on microscopic features of the system which are generally difficult to control, especially at the nanoscale. The use of a fundamentally different degree of freedom, which allows for the realization of memristive behavior, would be thus desirable.

In this work we demonstrate that such degree of freedom is provided by the electron spin and show that memristive behavior is common for the broad class of semiconductor spintronic devices. This class involves systems whose transport properties depend on the level of electron-spin polarization in a semiconductor, which is influenced by an external control parameter (such as an applied voltage). The adjustment in electron-spin polarization to a variation in the control parameter takes some time (this process involves diffusion and/or relaxation of electron-spin polarization), resulting in a memory effect accompanied by all other requirements for memristive behavior, such as absence of energy storage.

As an example of semiconductor spintronic system exhibiting memristive behavior, let us consider a semiconductor/ half-metal junction as schematically shown in Fig. 1(a). We

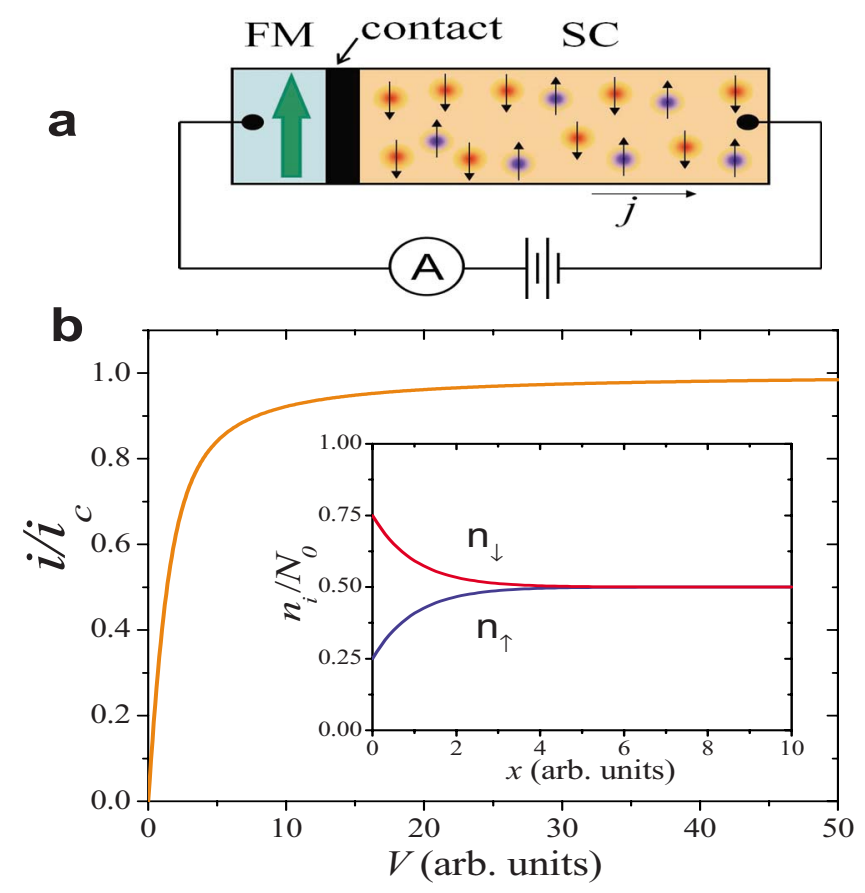

FIG. 1. (Color online) Semiconductor/half-metal junction. (a) Schematic representation of the circuit made of an interface between a semiconductor and a half metal. (b) Typical dc currentvoltage characteristics. Inset: spin-up and spin-down densities in the semiconductor region as a function of the distance from the contact. $i_{c}=e N_{0} \sqrt{D /\left(2 \tau_{s f}\right)}$ is the critical current density (Ref. 5). 
consider a junction with half metals (ferromagnets with $100 \%$ spin polarization at the Fermi level) because these act as perfect spin filters and, therefore, are more sensitive to the level of electron-spin polarization. However, we also expect that the prediction of memristive behavior is valid for junctions with ferromagnets having less than $100 \%$ spin polarization as a result of anticipated spin-based peculiarity in the $i$ - $V$ curve of these systems. ${ }^{4}$ Understanding the properties of the systems discussed in this Brief Report is of great interest in the context of actively studied spin-injection/spinextraction processes at semiconductor/ferromagnet junctions. ${ }^{4-14}$

We will be interested mainly in the process of spin extraction, where the electron flow is from the semiconductor into the half metal, which is especially interesting because of the recently predicted spin-blockade phenomenon in such junctions. ${ }^{4,5}$ The physics of the spin blockade is the following: the half metal accepts electrons of only one-say upspin direction. Spin-down electrons cannot enter the half metal and, therefore, form a cloud near the contact [see the inset in Fig. 1(b)] when a current flows through the system. This cloud increases with increasing current. At a critical current density the density of spin-up electrons near the contact becomes insufficient to provide a further current increase. In other words, transport of spin-up electrons through the contact becomes blocked by the cloud of spin-down electrons near the contact. It was predicted by the present authors ${ }^{4}$ that the spin blockade leads to a saturated $i$ - $V$ curve as that shown in Fig. 1(b). Here, we show that such a system has all the necessary components to exhibit memristive behavior.
In order to simulate current-voltage characteristics of the junction we assume that the applied voltage drops mainly on the semiconductor part and contact region. Taking a constant conductivity of the semiconductor region and contact conductivity proportional to spin-up electron density near the contact, ${ }^{4}$ we can write

$$
V=V_{s}+V_{c}=\left(\rho_{s} L+\rho_{c}^{0} \frac{N_{0}}{2 n_{\uparrow}(0)}\right) i,
$$

where $\rho_{s}$ and $L$ are the semiconductor resistivity and length, $\rho_{c}^{0}$ is the contact resistivity at $V \rightarrow 0, N_{0}$ is the electron density in the semiconductor, and $n_{\uparrow}(0)$ is the density of spin-up electrons near the contact. For simplicity, we assume constant electron density in the semiconductor, i.e., $n_{\uparrow}+n_{\downarrow}=N_{0}$.

The electron-spin densities in the semiconductor are described by the spin drift-diffusion model ${ }^{14}$

$$
\begin{gathered}
e \frac{\partial n_{\uparrow(\downarrow)}}{\partial t}=\operatorname{div} \vec{i}_{\uparrow(\downarrow)}+\frac{e}{2 \tau_{s f}}\left(n_{\downarrow(\uparrow)}-n_{\uparrow(\downarrow)}\right), \\
\vec{i}_{\uparrow(\downarrow)}=\sigma_{\uparrow(\downarrow)} \vec{E}+e D \nabla n_{\uparrow(\downarrow)} .
\end{gathered}
$$

Here, $\vec{i}_{\uparrow(\downarrow)}$ is the current density of spin-up (-down) electrons, $\tau_{s f}$ is the spin-relaxation time, $\sigma_{\uparrow(\downarrow)}$ is the conductivity of spin-up (-down) electrons, and $D$ is the diffusion coefficient.

According to the present classification scheme for memristive behavior, ${ }^{2}$ it is clear that the semiconductor/half-metal junction is a current-controlled memristive system. This can be seen by comparing directly Eqs. (4) and (5) with Eqs. (1) and (2), respectively [we can certainly write Eq. (5) in terms
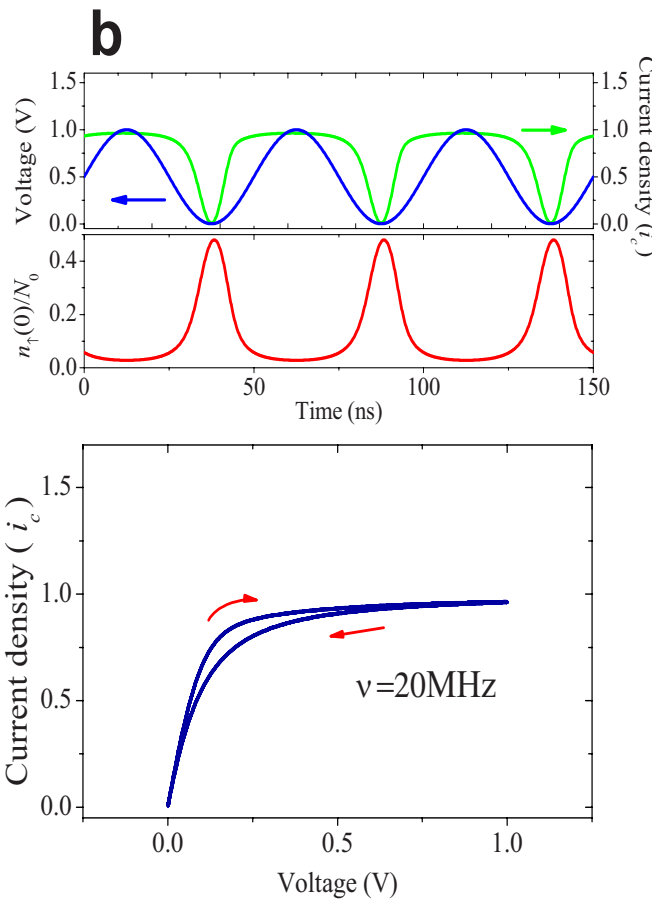

FIG. 2. (Color online) Simulations of ac response of the system. The applied voltages (blue lines) are $V=V_{1}+V_{2} \sin (2 \pi \nu t)$ with $V_{1}$ $=V_{2}=0.5 \mathrm{~V}, \nu=10^{9} \mathrm{~Hz}$ in (a) and $\nu=2 \cdot 10^{7} \mathrm{~Hz}$ in (b). In both cases the current densities (green lines in units of the critical current density) and spin-up electron densities near the contact (red lines) show saturation typical for spin blockade (Ref. 5). It is clearly seen that $i$ - $V$ hysteresis is significantly reduced in the low-frequency case. The calculations were made using the following system parameters: $L$ $=20 \mu \mathrm{m}, D=220 \mathrm{~cm}^{2} / \mathrm{s}, \mu=8500 \mathrm{~cm}^{2} /(\mathrm{V} \cdot \mathrm{s}), \tau_{s f}=2 \mathrm{~ns}, N_{0}=5 \times 10^{15} \mathrm{~cm}^{-3}$, and $\rho_{c}^{0} /\left(\rho_{s} L\right)=1$. 
of $n$ discrete variables]. In order to observe memristive behavior, we solved Eqs. (4)-(6) self-consistently at every given time ${ }^{15}$ with the following boundary conditions: $i_{\uparrow}(0)$ $=i, i_{\downarrow}(0)=0$, and $i_{\uparrow}(L)=i_{\downarrow}(L)=i / 2$ corresponding to the process of spin extraction at the contact.

Figure 2 shows results of our simulations with a timedependent applied voltage $V=V_{1}+V_{2} \sin (2 \pi \nu t)$ of high (1 $\mathrm{GHz}$ ) and low $(20 \mathrm{MHz})$ frequency $\nu$ with $V_{1} \geq V_{2}$. Such voltage profile has been selected in order to be always in the spin-extraction regime. The calculated $i-V$ curves (bottom panels in Fig. 2) exhibit a frequency-dependent hysteretic behavior typical of memristive systems. In particular, we can readily notice the distinctive zero-crossing property of these curves: no current flows through the structure when the voltage drop is zero ( $i-V$ curves pass through the $i=0, V=0$ point). This property is related to the fact that there is no energy storage in our system as opposed to the energy storage in the usual capacitive or inductive circuit elements.

Another interesting feature of the $i$ - $V$ curves is their frequency behavior. It follows from Fig. 2 that the hysteresis is significantly suppressed at $20 \mathrm{MHz}$ frequency. This is a manifestation of the fact that at low frequencies our system behaves essentially as a nonlinear resistor. Physically, at low applied voltage frequencies, the electron-spin polarization in the semiconductor has enough time to adjust to any present value of the voltage. Therefore, the current through the system at low frequencies is nonlinear (because of spin blockade) but essentially history independent (no hysteresis). It is also clear that at very high frequencies $(\nu \rightarrow \infty)$ the electronspin polarization does not have any time for redistribution within the oscillation period. Therefore, the spin-up density near the contact and, correspondingly, the contact resistivity can be considered constant, so that the device operates as a linear resistor. The above described frequency behavior of the semiconductor/half-metal junction is typical for memristive systems. ${ }^{2}$

Although the model represented by Eqs. (4)-(6) takes into account the main physics of the underlying system operation, it is still quite complex for circuit analysis. Here, we thus discuss a simplified (and more transparent) model which can be obtained in the following way. Instead of tracking the whole spin-density distribution in the semiconductor, let us focus our attention on the integrated spin density (surface spin density) $N_{\uparrow(\downarrow)}=\int_{0}^{L} n_{\uparrow(\downarrow)} d x$ and select this quantity as the only system parameter. Integrating Eq. (5) from 0 to $L$ (i.e., over the whole semiconductor length) we obtain the following equation:

$$
e \frac{\partial N_{\uparrow}}{\partial t}=-\frac{1}{2} i+\frac{e}{\tau_{s f}}\left(\frac{N L}{2}-N_{\uparrow}\right) .
$$

Equation (7) simply states that the change in the integrated spin-up density is due to the injection/extraction of spin-up electrons through the semiconductor boundaries [the first "current" term in the right-hand side (RHS) of Eq. (7)] and spin-relaxation processes [the second term in the RHS of Eq. (7)]. Although the knowledge of $N_{\uparrow}$ is not sufficient to exactly obtain $n_{\uparrow}(0)$, which enters into Eq. (4), we can approximately write
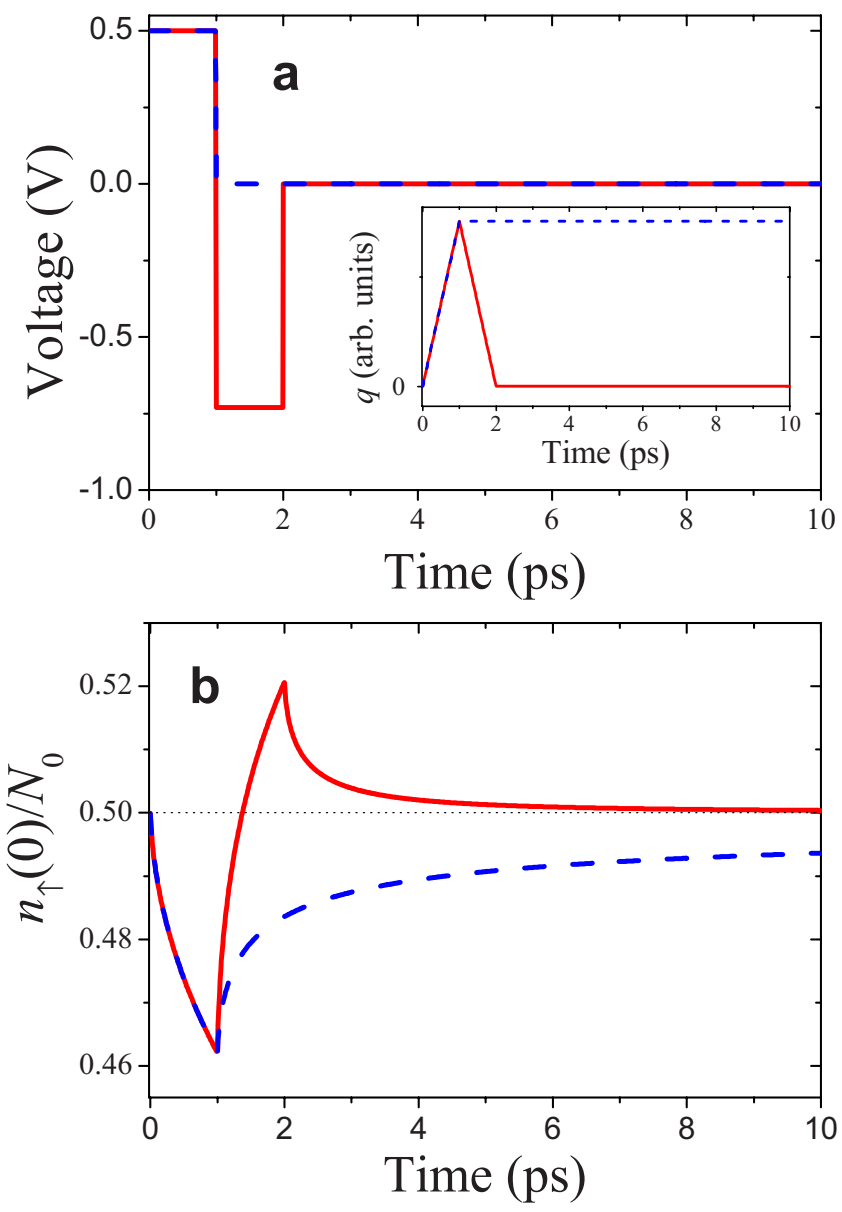

FIG. 3. (Color online) System dynamics excited by step voltages. (a) Unipolar (blue line) and bipolar (red line) step voltage profiles used in our calculations. The spin-injection process (at negative applied voltage) was modeled using a constant interface resistance model. Inset: Total charge flown through the system as a function of time. The profile of bipolar voltage was selected in such a way that the corresponding total charge $q=0$ for $t>2$ ps (see the red line). (b) Evolution of spin-up electron density near the interface. In the case of bipolar voltage excitation (red line), the final value of spin-up density is close to its initial value (shown by the dotted line). This is a manifestation of a nearly perfect memristor behavior.

$$
n_{\uparrow}(0)=g\left(N_{\uparrow}\right) \text {, }
$$

where $g$ is a given smooth function. The simplified circuit model given by Eqs. (4), (7), and (8) describes again a memristive system. However, at short times, when spin-relaxation processes are not important the last term in the RHS of Eq. (7) can be neglected. The resulting set of equations describes then a perfect memristor. Indeed, integrating Eq. (7) we obtain

$$
N_{\uparrow}(t)=N_{\uparrow}(0)-\frac{1}{2 e} \int_{0}^{t} i(\tau) d \tau=N_{\uparrow}(0)-\frac{1}{2 e} q(t),
$$

and, substituting Eqs. (8) and (9) into Eq. (4), we get 


$$
V=\left[\rho_{s} L+\rho_{c}^{0} \frac{N_{0}}{2 g\left(N_{\uparrow}(0)-\frac{1}{2 e} q(t)\right)}\right] .
$$

It follows from Eq. (10), which is a particular case of Eq. (3), that the resistivity of the system depends only on the amount of charge $q(t)$ flown through it.

In order to test the simplified circuit model predictions, we calculate, using Eqs. (4)-(6), the short-time system response to unipolar and bipolar voltage step excitations. Figure 3(b) demonstrates that our structure behaves almost as a perfect memristor at short times. In particular, the red line in Fig. 3(b) shows that when the total charge flown through the structure is equal to zero, the value of the system parameter $n_{\uparrow}(0)$ (and consequently of the device resistivity) becomes very close to its initial value already at $t=5 \mathrm{ps}$. The opposite behavior is demonstrated by the blue line in Fig. 3(b): when the total charge flowing in the system is different from zero, the value of the spin-up density at the interface $n_{\uparrow}(0)$ is quite different from its initial value even at $t=10 \mathrm{ps}$.

Even though the focus of this Brief Report is on the physical mechanisms underlying the spin memristive behavior, we briefly discuss possible applications of these results. Certainly, since the spin-relaxation time in semiconductors is quite short, the use of spin memristive systems in memory applications is unlikely. The problem is that the refreshing rate for spin memristive systems should be much higher than those in usual dynamic random access memory (64 ms) unless a system with much longer spin lifetime is used. Instead, a more promising application area is analog electronics, in which utilization of spin memristive systems may be beneficial because of their unique characteristics. Finally, another interesting direction is in solid-state modeling and realization of neural networks and mechanisms of learning.

To summarize, we have demonstrated that a semiconductor/half-metal junction is in fact a memristive system. The origin of its unusual behavior in a circuit is completely based on the electronic spin degree of freedom, which is much easier to control than ionic transport at the nanoscale. In our scheme, the principal role is played by electron-spin diffusion and relaxation processes which drive the system to equilibrium. Moreover, we would like to note that the spin memristive behavior is not limited to the discussed structure; it should be typical of many semiconductor spintronic devices that involve spin filters, general semiconductor-ferromagnet junctions, etc. We also expect the memristive behavior to be more pronounced in structures with low electron density, where the level of electron-spin polarization can be significantly varied by external control parameters. We thus believe that our demonstration of memristive effects in spintronics is an important step forward in future practical applications of the newly discovered fourth circuit element.

This work was partially supported by the National Science Foundation and Department of Energy.
${ }^{1}$ L. O. Chua, IEEE Trans. Circuit Theory 18, 507 (1971).

${ }^{2}$ L. O. Chua and S. M. Kang, Proc. IEEE 64, 209 (1976).

${ }^{3}$ D. B. Strukov, G. S. Snider, D. R. Stewart, and R. S. Williams, Nature (London) 453, 80 (2008); J. J. Yang, M. D. Pickett, X. Li, D. A. A. Ohlberg, D. R. Stewart, and R. S. Williams, Nat. Nanotechnol. 3, 429 (2008).

${ }^{4}$ Yu. V. Pershin and M. Di Ventra, Phys. Rev. B 77, 073301 (2008).

${ }^{5}$ Yu. V. Pershin and M. Di Ventra, Phys. Rev. B 75, 193301 (2007).

${ }^{6}$ E. I. Rashba, Eur. Phys. J. B 29, 513 (2002).

${ }^{7}$ J. D. Albrecht and D. L. Smith, Phys. Rev. B 68, 035340 (2003).

${ }^{8}$ I. Žutić, J. Fabian, and S. Das Sarma, Phys. Rev. Lett. 88,
066603 (2002).

${ }^{9}$ R. K. Kawakami, Y. Kato, M. Hanson, I. Malajovich, J. M. Stephens, E. Johnston-Halperin, G. Salis, A. C. Gossard, and D. D. Awschalom, Science 294, 131 (2001).

${ }^{10}$ S. A. Crooker, M. Furis, X. Lou, C. Adelmann, D. L. Smith, C. J. Palmstrom, and P. A. Crowell, Science 309, 2191 (2005).

${ }^{11}$ M. Shen, S. Saikin, and M.-C. Cheng, J. Appl. Phys. 96, 4319 (2004).

${ }^{12}$ Y. Y. Wang and M. W. Wu, Phys. Rev. B 72, 153301 (2005).

${ }^{13}$ H. Dery and L. J. Sham, Phys. Rev. Lett. 98, 046602 (2007).

${ }^{14}$ Z. G. Yu and M. E. Flatté, Phys. Rev. B 66, 201202(R) (2002).

${ }^{15}$ D. L. Scharfetter and H. K. Gummel, IEEE Trans. Electron Devices ED-16, 64 (1969). 